\title{
ASPECTOS MORFOLÓGICOS DA PROBÓSCIDE DE MUSCOIDEA (DIPTERA, MUSCOMORPHA) ${ }^{1}$
}

\author{
Sônia Maria Prevedello Coelho ${ }^{2}$
}

\begin{abstract}
MorphologicAl aspectS OF THE PROBOSCIS OF Muscoidea (DiPTERA, MUSCOMORPHA). A study comparing the morphology of the proboscis of the following muscoid species is presented: Scatophaga stercoraria (Linnaeus, 1758) (Scatophagidae), Fannia canicularis (Linnaeus, 1761) (Fanniidae), Taenomyia auricollis Stein, 1918 (Anthomyiidae) and Phaonia valida (Harris, 1780) (Muscidae). Comparisons were made with Archiborborus sp. (Acalyptratae, Sphaeroceridae), Calliphora vicina Robineau-Desvoidy, 1830 (Oestroidea, Calliphoridae) and other muscids.

KEY WORDS. Calliphoridae, Muscoidea, Sphaeroceridae, morphology, proboscis
\end{abstract}

O aparelho bucal nos Diptera é primariamente adaptado para sugar líquidos ou alimentos liquefeitos. Há, no entanto, uma grande variabilidade nas estruturas da probóscide, tanto em morfologia como em função (MCALPINE 1981).

De modo geral, os Nematocera apresentam as peças bucais modificadas em estiletes picadores e nos Muscomorpha, os estiletes foram perdidos e as peças bucais estão adaptadas a absorver alimento liquefeito (BOUDREAUX 1987).

Em alguns muscóideos, como Stomoxys Geoffroy, 1762 e Glossina Wiedemann, 1830, foi readquirida a habilidade de picar e sugar através do lábio, o qual transformou-se em uma estrutura picadora (BOUDREAUX 1987).

$\mathrm{Na}$ maioria das espécies da ordem, o alimento é ingerido pela aplicação da labela em substâncias líquidas como o néctar, ou em alimentos secos como o grão de pólen, o qual é primeiramente liquefeito com a saliva (MCALPINE 1981).

PETERSON (1916) estudou as peças bucais dos Diptera, utilizando um tipo hipotético para determinar como as peças bucais se modificaram e comentou que o lábio é a estrutura mais especializada e característica do aparelho bucal.

A maioria dos estudos de probóscide está relacionada a espécies de Diptera, que apresentam interesse médico-veterinário [veja as referências bibliográficas de MATSUDA (1965) e MCALPINE (1981)].

Deste modo, neste trabalho foi feito um estudo comparativo de alguns aspectos da probóscide de representantes de Muscoidea, Scatophaga stercoraria (Linnaeus, 1758) (Scatophagidae), Fannia canicularis (Linnaeus, 1761) (Fanniidae), Taenomyia auricollis Stein, 1918 (Anthomyiidae) e Phaonia valida (Harris, 1780) (Muscidae), os quais foram comparados com Archiborborus sp. (Acalyptra-

1) Contribuição número 923 do Departamento de Zoologia, Universidade Federal do Paraná.

2) Departamento de Zoologia, Universidade Federal do Paraná. Caixa Postal 19020. 81531-990 Curitiba, Paraná, Brasil. 
tae, Sphaeroceridae), Calliphora vicina Robineau-Desvoidy, 1830 (Oestroidea, Calliphoridae) e outras espécies de Muscidae estudadas por outros autores.

\section{MATERIAL E MÉTODOS}

Foi utilizado material proveniente da Coleção de Entomologia Pe. Jesus S. Moure, Departamento de Zoologia, Universidade Federal do Paraná, Curitiba, Brasil (DZUP) (responsável: Dr. C.J.B. de Carvalho).

Foram dissecadas as probóscides de um exemplar fêmea das seguintes espécies: Scatophaga stercoraria, Fannia canicularis, Taenomyia auricollis, Phaonia valida, Archiborborus sp. e Calliphora vicina.

A metodologia para dissecação e diafanização é a mesma utilizada por CARVAlHo (1989a).

A terminologia adotada segue BlETChLY (1953) e CARVALHO (1989a), exceto para o esclerito localizado no haustelo, onde se utilizou o termo premento ao invés de mento (PETERSON 1916; MCALPINE 1981).

\section{RESULTADOS E DISCUSSÃO}

A probóscide de $S$. stercoraria (Muscidae) foi descrita minuciosamente por BLETCHLY (1953).

Assim foi feita apenas uma sinopse da probóscide de $S$. stercoraria, a única com hábito predador entre as espécies estudadas e com os escleritos bem desenvolvidos, para posteriormente ser feita uma comparação com outras espécies estudadas e citadas na literatura.

A probóscide está dividida em três regiões: basiprobóscide ou rostro; medioprobóscide ou haustelo e distiprobóscide ou labela (PETERSON 1916; MATSUDA 1965; HENNIG 1973).

Em S. stercoraria (Fig. 1) observou-se que o rostro é uma região em forma de cone, provida de áreas membranosas e esclerotinizadas. Em vista lateral, há dois amplos escleritos: o fulcro e o clípeo.

O fulcro é maior e menos esclerotinizado do que o clípeo e apresenta um arco anterior maior e um posterior menor.

O clípeo, na região posterior, apresenta lateralmente uma leve ponta.

Outra estrutura que faz parte do rostro é o esclerito hióide. Em vista lateral, este esclerito é pequeno e em forma de lua crescente, que localiza-se abaixo do arco posterior do fulcro e mais internamente, articulando-se com o labro-epifaringe.

Fronto-lateralmente localiza-se um par de palpos maxilares e na região posterior do fulcro, um par de apódemas.

Há três músculos associados ao apódema, que auxiliam na extensão e flexão do haustelo (BLETCHLY 1953).

Na região mediana da probóscide observa-se o haustelo. 


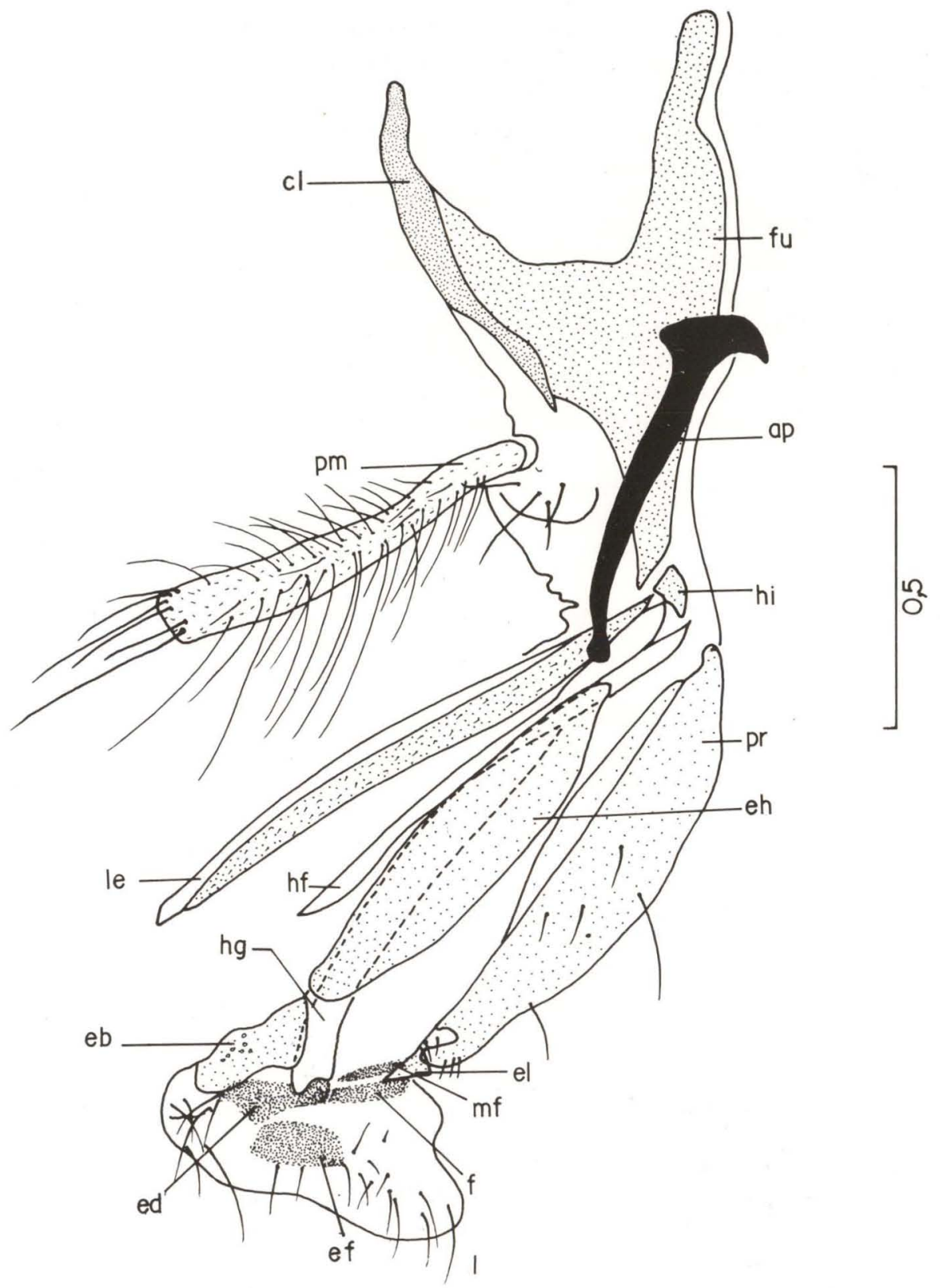

Fig. 1 Probóscide da fêmea de Scatophaga stercoraria, vista lateral. (ap) Apódema, (cl) clípeo, (eb) esclerito basal, (ed) esclerito discal, (ef) epifurca, (eh) esclerito dorsal do haustelo, (el) esclerito lateral, (f) furca, (fu) fulcro, (hf) hipofaringe, (hg) hipoglossa, (hi) esclerito hióide, (le) labro-epifaringe, (mf) faixa mento-furcal, (pm) palpo maxilar, (pr) premento. Escala em milímetros. 
Ventro-lateralmente, há uma grande estrutura esclerotinizada protegendo todo o haustelo, denominada premento. Também é denominada teca ou mento por alguns autores (PETERSON 1916; BLETCHLY 1953).

Dorso-lateralmente observa-se o esclerito dorsal do haustelo e na região postero-lateral do premento, o esclerito lateral.

Na região dorsal do haustelo ocorrem ainda as estruturas: labro-epifaringe, hipofaringe e hipoglossa.

Finalmente, na parte apical da probóscide, localiza-se a labela, que é reduzida e com poucas pseudotraquéias.

$\mathrm{Na}$ labela ocorrem ainda as estruturas: esclerito basal dorsalmente, esclerito discal latero-dorsalmente e a faixa mento-furcal, epifurca e furca internamente.

Nos Muscomorpha o clípeo é reduzido e em forma de "U" invertido, o qual está separado da margem inferior da face pela membrana fronto-clipeal (MCALPINE 1981).

Em Muscidae, o clípeo, na região posterior, apresenta lateralmente uma ponta, que pode ser forte, ultrapassando bastante o fulcro, ou leve, pouco ultrapassando o fulcro, caráter que foi utilizado por CARVALHO (1989c) na análise cladística de Muscidae.

CARValho (1993) definiu o monofiletismo do gênero Dolichophaonia Carvalho, 1993 (Muscidae, Phaoniinae) através de dois caracteres do clípeo: lateralmente, na região anterior com esclerotinização saliente que ultrapassa o fulcro e na região posterior, com forte ponta em forma de gancho.

O fulcro apresenta um arco posterior, o qual é variável com relação ao seu comprimento, caráter que também foi utilizado por CARVALHO (1989b) na análise cladística de Muscidae.

O esclerito hióide, segundo MCALPINE (1981), está associado à bomba cibarial, na base do canal alimentar.

MATSUDA (1965) comentou que o esclerito hióide pode ser um vestígio da mandíbula, a qual era considerada ausente nos Muscomorpha.

Observou-se que o haustelo é mais esclerotinizado nas espécies predadoras do que as que não possuem hábito predador.

CARVAlHo (1989c) utilizou o caráter haustelo na análise cladística de Muscidae. Este autor hipotetizou que o haustelo começa a esclerotinizar-se nos ramos basais. Nos grupos predadores ocorre um aumento de esclerotinização do premento até atingir uma total esclerotinização, para posteriormente ficar parcialmente esclerotinizado nos ramos apicais. labiais.

A labela, segundo MCALPINE (1981), é uma modificação do par de palpos

Comparando-se a probóscide de S. stercoraria com a de Coenosia tigrina (Fabricius, 1775) (Muscidae), espécie predadora estudada por KUHNE (1992), observou-se que as regiões que sofreram maiores modificações são o haustelo e a labela.

KUHNE (1992) citou três escleritos na região dorsal do haustelo, sendo que o segundo esclerito é provavelmente homólogo ao esclerito dorsal do haustelo. 
A labela de C. tigrina é mais reduzida do que a de $S$. stercoraria e apresenta um quarto esclerito que provavelmente é homólogo ao esclerito basal.

KUHNE (1992) comentou que há uma redução no número de dentes prestomais e pseudotraquéias em C. tigrina, mostrando uma extrema diferença e especialização entre as partes esclerotinizadas da labela.

CARVALHO (1989c) desenhou as probóscides de Limnophora aurifascies Stein, 1911 e Neodexiopsis sp. representantes de Coenosiinae (Muscidae). Este autor comentou que a redução da labela nestas espécies é uma sinapomorfia para a subfamília.

Entretanto, foi observado que em L. aurifascies há provavelmente uma fusão dos escleritos dorsais com o premento e em Neodexiopsis sp., os escleritos dorsais estão individualizados como ocorre em C. tigrina.

O mesmo padrão do haustelo que ocorre em $L$. aurifascies, foi observado em Parvomusca paula Medeiros, 1980 e em Hypsomyia nebulicola Couri \& Carvalho, 1994, espécies pertencentes a Coenosiinae (Muscidae), as quais foram ilustradas por COURI \& CARVAlHO $(1993,1994)$.

Nas espécies que não possuem hábito predador, há um aumento de áreas membranosas, desenvolvimento da labela e das pseudotraquéias.

O esclerito dorsal do haustelo e o esclerito basal geralmente estão presentes, mas reduzidos em relação às espécies predadoras, e o esclerito lateral e os outros escleritos dorsais observados por KUHNE (1992) em C. tigrina, estão ausentes.

Em Archiborborus sp. (Fig. 2), representante de Sphaeroceridae (Schizophora, Acallyptratae), o esclerito dorsal do haustelo e o esclerito basal são bem desenvolvidos e providos de muitos cílios.

Nos representantes de Muscoidea, sensu MCALPINE (1981), estudados, observou-se uma redução do esclerito dorsal do haustelo e diminuição dos cílios tanto no esclerito dorsal do haustelo como no esclerito basal. Em Fannia canicularis (Fig. 3) o esclerito dorsal do haustelo e o esclerito basal são mais desenvolvidos do que em Taenomyia auricollis (Fig. 4) e Phaonia valida (Fig. 5).

Apesar de MCALPINE (1989) considerar Fanniidae como grupo-irmão de Muscidae, através da análise das probóscides, observou-se que provavelmente Anthomyiidae é mais próxima de Muscidae do que Fanniidae, corroborando-se assim a idéia de MiCHELSEN (1991) que Anthomyiidae é grupo-irmão de Muscidae.

Estudou-se também, a probóscide de Calliphora vicina (Oestroidea, Calliphoridae) (Fig. 6) para observar o padrão do esclerito dorsal do haustelo e do esclerito basal nos ramos mais apicais de Calyptratae. Notou-se que somente o esclerito basal está presente e que o haustelo dorsalmente, é totalmente membranoso.

CARvalho (1989a,b,c,d, 1993) estudou as probóscides de outras espécies de Muscidae e só ilustrou o esclerito basal de algumas espécies. Este autor também desenhou as probóscides de algumas espécies de Palpibracus Rondani, 1864 (Muscidae, Azeliinae), onde observa-se que o esclerito dorsal do haustelo e o esclerito basal são desenvolvidos e apresentam muitos cílios. 

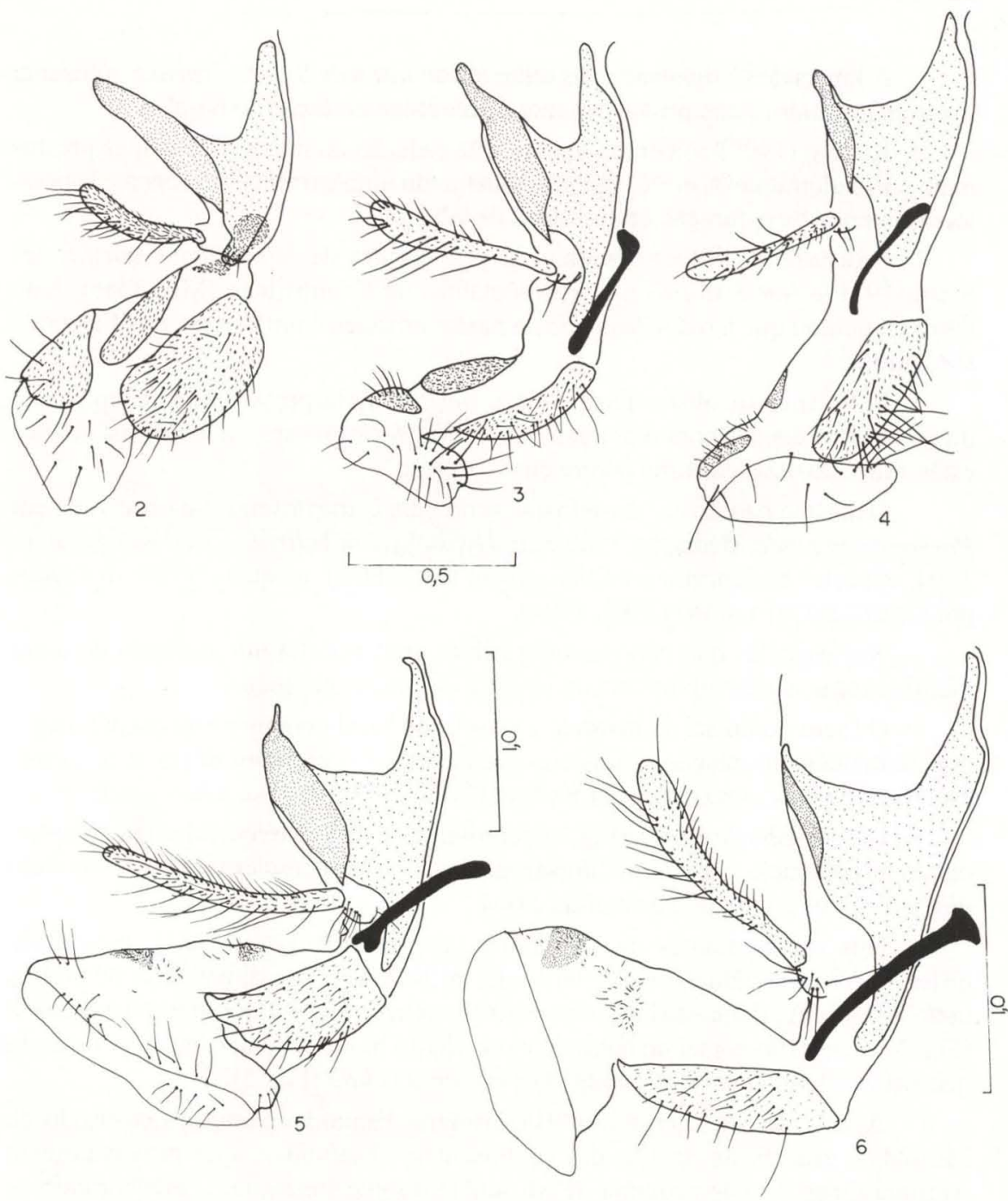

Figs 2-6. Probóscide da fêmea, vista lateral. (2) Archiborborus sp.; (3) Fannia canicularis; (4) Taenomyia auricollis; (5) Phaonia valida; (6) Calliphora vicina. Escalas em milimetros.

Na probóscide de Scutellomusca scutellaris (Fabricius, 1805) (Muscidae, Mydaeinae), espécie estudada por Couri \& CARVALHo (1992), observou-se o mesmo padrão do haustelo que ocorre em Palpibracus, exceto na redução de cílios nos escleritos.

CARVAlHo \& COURI (1992) representaram a probóscide de Agenamyia exotica Carvalho \& Couri, 1992 (Muscidae, Coenosiinae), onde observa-se que o esclerito dorsal do haustelo é desenvolvido, mas não apresenta cílios e o esclerito basal é pequeno e com poucos cílios. 
Outros escleritos observados em espécies de Coenosiinae como C. tigrina e Neodexiopsis sp., citadas anteriormente, não foram ilustrados em $A$. exotica por CARVALHO \& COURI (1992).

COURI \& PAMPLONA (1992) e MOTTA \& COURI (1995) também estudaram as probóscides de espécies de Muscidae, mas não ilustraram os escleritos dorsais do haustelo e da labela.

CARVAlHo \& COURI (1993) desenharam a probóscide de Itatingamyia bivittata Albuquerque, 1979 (Muscidae, Azeliinae), entretanto não foi possível a interpretação dos escleritos.

\section{CONCLUSÕES}

Nas espécies com hábito predador como S. stercoraria, L. aurifascies, Neodexiopsis sp., C. tigrina, $P$. paula e $H$. nebulicola (Muscidae) há um aumento da esclerotinização do haustelo, diminuição das áreas membranosas e redução da labela.

O esclerito basal está presente em todas as espécies estudadas: Archiborborus sp., S. stercoraria, F. canicularis, T. auricollis, P. valida e C. vicina.

$\mathrm{O}$ esclerito dorsal do haustelo e o esclerito basal são geralmente mais devenvolvidos e com mais cílios nas espécies dos ramos mais basais de Schizophora, como Archiborborus sp. e $S$. stercoraria, e diminui nas espécies dos ramos mais apicais, até que em $C$. vicina o esclerito basal está ausente.

Com o padrão observado no esclerito dorsal do haustelo e no esclerito basal das espécies de Muscoidea estudadas, é provável que a hipótese de que Anthomyiidae seja grupo-irmão de Muscidae, como hipotetizado por MICHELSEN (1991), seja verdadeira.

O tamanho da labela, caráter utilizado por CARVALHO (1989b) na análise cladística de Muscidae, provavelmente posiciona a espécie C. tigrina nos Coenosiinae.

AGRADECIMENTOS. Ao Prof. Dr. Claudio José Barros de Carvalho pela orientação.

\section{REFERÊNCIAS BIBLIOGRÁFICAS}

BLETCHLY, J.D. 1953. The mouth-parts of the yellow dung-fly, Scopeuma (= Scatophaga) stercoraria (L.) (Diptera, Cordyluridae). Proc. Zool. Soc. London 123 (1): 143-165.

BOUDREAUX, H.B. 1987. Arthropod phylogeny with special reference to insects.

Malabar, Florida, Robert E. Krieger Publishing Company, 319p.

CARValho, C.J.B. DE. 1989a. Revisão das espécies e posição sistemática de Palpibracus Rondani (Diptera, Muscidae). Revta bras. Zool. 6 (2): 325-376. 1989b. Revisão de Psilochaeta Stein e descrição de Dalcyella gen. n. do

Chile (Diptera, Muscidae). Revta bras. Zool. 6 (3): 485-506. 1989c. Classificação de Muscidae (Diptera): uma proposta através da 
análise cladística. Revta bras. Zool. 6 (4): 627-648.

. 1989d. Classificação de Phaonia Robineau-Desvoidy (Diptera, Muscidae) da Região Neotropical. I. Considerações sobre a sinonímia de Bigotomyia Malloch, com descrição de uma espécie nova. Mem. Inst. Oswaldo Cruz 84 (4): 87-90.

1993. Dolichophaonia, gen.n. (Diptera, Muscidae, Phaoniinae): descrições, novas combinações, sinonímias e chave para as espécies. Revta bras. Ent. 37 (1): 19-34.

CARVAlHO, C.J.B. DE \& M.S. Couri. 1992. Descrição de Agenamyia exotica, sp.n. (Diptera, Muscidae), chave para as espécies do gênero e considerações sobre sua posição sistemática. Revta bras. Ent. 36 (1): 21-24.

1993. Itatingamyia Albuquerque, 1979: redescrição do gênero, considerações sobre sua posição sistemática e descrição da terminália do macho e da fêmea de I. bivittata (Diptera, Muscidae). Revta bras. Ent. 37 (3): 591594.

Couri, M.S. \& C.J.B. DE CARVALHO. 1992. Sobre a identidade de Hemixanthomya marginata Albuquerque, 1954 e considerações sobre a posição sistemática de Scutellomusca Townsend, 1931 (Diptera, Muscidae). Rev. Brasil. Biol. 52 (1): 93-97.

1993. Parvomusca Medeiros, 1980: redescrição do gênero e descrição de uma espécie nova (Diptera, Muscidae). Revta bras. Ent. 37 (4): 731-735.

1994. Hypsomyia nebulicola, gen.n., sp.n. de Limnophorini do Pico da Neblina, Brasil (Diptera, Muscidae, Coenosiinae). Rev. Brasil. Biol. 54 (4): 569-573.

Couri, M.S. \& D. PAmplonA. 1992. Cordiluroides Albuquerque, 1954 (Diptera, Muscidae, Coenosiinae): redescrições, notas, sinonímia e chave para as espécies. Revta bras. Ent. 36 (2): 255-262.

HENNIG, W. 1973. Ordnung Diptera (Zweiflügler). Handb. Zool. 4 (2) 2/31 (Lfg. 20): 1-337.

KuHNE, K-ST. 1992. Morphologische Beschreibung und Funktionsmechanismus des Proboscis von Coenosia tigrina Fab. (Muscidae). Zool. Beitr. N.F. 34 (1): 113-125.

Matsuda, R. 1965. Morphology and evolution of the insect head. Mem. Amer. ent. Inst. 4: 1-334.

MCALPINE, J.R. 1981. Morphology and terminology, p.9-63. In: J.F. MCALPINE; B.V.PETERSON; G.E. SHEWELL; H.J. TESKEY; J.R. VOCKEROTH \& D.M. WOOD (Eds). Manual of Neartic Diptera. Otawa, Agriculture Canada, Research Branch Monograph 27, vol. 1, VI+674p.

1989. Phylogeny and classification of the Muscomorpha. p. 1397-1518. In: J.F. MCALPINE \& D.M. Wood (Eds). Manual of Neartic Diptera. Otawa, Agriculture Canada, Research Branch Monograph 32, vol. 3, VI+1581p.

MiChelsEN, V. 1991. Revision of the aberrant New World genus Coenosopsia (Diptera, Anthomyiidae), with a discussion of anthomyiid relationships. Syst. Entomol. 16 (1): 85-104. 
MotTA, H.C.G. \& M.S. CouRI. 1995. Redescrição de Bithoracochaeta Stein (Diptera, Muscidae) com comentários sobre sua posição sistemática. Revta bras. Zool. 12 (1): 47-53.

PETERSON, A. 1916. The head-capsule and mouth-parts of Diptera. Illinois biol. Monogr. 3 (2): 1-113.

Recebido em 10.I.1996; aceito em 20.V.1997 